\title{
Many-Molecule Reaction Triggered by a Single Photon in Polaritonic Chemistry
}

\author{
Javier Galego, ${ }^{1}$ Francisco J. Garcia-Vidal, ${ }^{1,2, *}$ and Johannes Feist ${ }^{1, \dagger}$ \\ ${ }^{1}$ Departamento de Física Teórica de la Materia Condensada and Condensed Matter Physics Center (IFIMAC), \\ Universidad Autónoma de Madrid, E-28049 Madrid, Spain \\ ${ }^{2}$ Donostia International Physics Center (DIPC), E-20018 Donostia/San Sebastian, Spain
}

(Received 18 May 2017; published 27 September 2017)

\begin{abstract}
The second law of photochemistry states that, in most cases, no more than one molecule is activated for an excited-state reaction for each photon absorbed by a collection of molecules. In this Letter, we demonstrate that it is possible to trigger a many-molecule reaction using only one photon by strongly coupling the molecular ensemble to a confined light mode. The collective nature of the resulting hybrid states of the system (the so-called polaritons) leads to the formation of a polaritonic "supermolecule" involving the degrees of freedom of all molecules, opening a reaction path on which all involved molecules undergo a chemical transformation. We theoretically investigate the system conditions for this effect to take place and be enhanced.
\end{abstract}

DOI: 10.1103/PhysRevLett.119.136001

Photochemical reactions underlie many essential biological functions, such as vision or photosynthesis. In this context, the second law of photochemistry (also known as the Stark-Einstein law) states that "one quantum of light is absorbed per molecule of absorbing and reacting substance" [1]. This means that the quantum yield $\phi=$ $N_{\text {prod }} / N_{\text {phot }}$ of the reaction, which describes the percentage of molecules that end up in the desired reaction product per absorbed photon, has a maximum value of 1 . This limit can be overcome in some specific cases, such as in photochemically induced chain reactions [2-4], or in systems that support singlet fission to create multiple triplet excitons (and thus electron-hole pairs) in solar cells $[5,6]$.

Polaritonic chemistry, i.e., the potential to manipulate chemical structure and reactions through the formation of polaritons (hybrid light-matter states) was experimentally demonstrated in 2012 [7], and has become a topic of intense experimental and theoretical research in the past few years [8-18]. However, existing applications and proposals have been limited to enhancing or suppressing the rates of single-molecule reactions. In this Letter we demonstrate a novel and general approach to circumvent the StarkEinstein law by exploiting the collective nature of polaritons formed by bringing a collection of molecules into strong coupling with a confined light mode. We show that this can allow many molecules to undergo a photochemical reaction after excitation by just a single photon. In contrast to conventional chain reactions, this polaritonic reaction does not rely on a near-resonant energy transfer condition between the excited stable and metastable ground state in the molecule. Our finding illustrates how polaritonic chemistry can open fundamentally new pathways that allow for reactions that are not present in the uncoupled system, and thus possesses the potential to unlock a new class of collective reactions.
The mechanism we introduce relies on the delocalized character of the hybrid light-matter excitations (polaritons) formed under strong coupling (SC), which conceptually leads to the formation of a single "supermolecule" involving all the molecules as well as the trapped photon. The photochemical reaction induced in this supermolecule can then lead to most or even all of its molecular constituents undergoing a structural change, corresponding to an effective quantum yield significantly larger than 1 , although it can obviously not lead to a violation of energy conservation. We thus investigate a class of reactions that release energy, i.e., where the initial starting state after absorption of a photon has higher energy than the final state, in which all involved molecules have undergone a reaction. We focus on a class of model molecules with a structure as proposed for use in solar energy storage [19-21], described within a simplified model treating a single reaction coordinate, as shown in Fig. 1(a). In our model molecule, the potential energy surface (PES) associated with the electronic ground state contains two local minima: a stable ground-state configuration (at $q=q_{\mathrm{s}} \approx 0.8$ a.u.) and a metastable configuration (at $q=q_{\mathrm{ms}} \approx-0.7$ a.u.) that contains a stored energy of about $1 \mathrm{eV}$. The activation barrier for thermal relaxation from the metastable configuration to the global minimum has a height of more than $1 \mathrm{eV}$, leading to a lifetime on the order of days or even years for the metastable configuration [22], and thus making it interesting for solar energy storage. In addition, the molecule possesses an excited-state PES with a relatively flat minimum close to the ground transition state. The decay from the excited-state to the ground-state PES in the bare molecule is taken to be relatively slow, and is represented by purely radiative decay in our calculations for simplicity (although this assumption is not essential, see Sec. I of the Supplemental Material [23]). As indicated in Fig. 1(a), fast 
vibrational relaxation on the excited-state surface and subsequent radiative decay from the excited wave packet in thermal equilibrium then gives a roughly equal reaction quantum yield for reaching either the stable (44\%) or the metastable configuration (56\%) in the electronic ground state (see Sec. I of the Supplemental Material for more details [23]). As expected in a conventional photochemical reaction, the quantum yields in the bare molecule add up to 1 (indeed, the Stark-Einstein law can be reformulated as "the sum of quantum yields must be unity").

Let us first describe the molecular model in more detail. The two adiabatic PESs (ground state and first excited state) of the single bare molecule are both constructed independently from two coupled harmonic potentials as follows:

$V_{i}(q)=\frac{1}{2}\left\{v_{i}(q)+w_{i}(q)-\sqrt{4 h_{i}^{2}+\left[v_{i}(q)-w_{i}(q)\right]^{2}}\right\}$,

where $i \in\{g, e\}$ indicates either the ground-state or excited-state PES. The terms $v_{i}(q)$ and $w_{i}(q)$, coupled through $h_{i}$, are harmonic (i.e., quadratic) potentials. While there is a relatively large number of free parameters that control the molecular structure, we have checked that the results presented below are insensitive to small variations as long as the general shape of the PES is maintained. More details and the exact parameters used are given in Sec. II of the Supplemental Material [23].

We now assume that a collection of these molecules is placed inside a photonic structure supporting a single confined light mode. This could be physically realized using a variety of approaches, such as dielectric microcavities, photonic crystal cavities, or plasmonic nanocavities [26-32]. We analyze the system using the methods developed in our previous work $[9,10]$, which describe the collective structure of the coupled system of many molecules and the photon mode through the use of polaritonic PES encompassing the nuclear degrees of freedom of all molecules. This picture immediately allows us to take into account and understand the collective motion induced on the molecules through their mutual coupling to the photonic mode. For the specific case of five molecules, we show the coupled PESs in Fig. 1(b). Here, we take a cut of the five-dimensional PES where only the first molecule $\left(q_{1}\right)$ is allowed to move, while all others are fixed to the equilibrium position of the metastable ground-state configuration $\left(q_{i}=q_{\mathrm{ms}}\right.$ for $\left.i=2, \ldots, 5\right)$.

When introducing the quantized light mode to the system, its coupling to the molecules is accounted for through an additional term in the Hamiltonian, given by the scalar product of the molecular dipole operator $\hat{\vec{\mu}}(q)=$ $\vec{\mu}_{e g}(q)\left(\sigma^{\dagger}+\sigma\right)$, where $\sigma=|g\rangle\langle e|$ annihilates an electronic excitation, and the electric field of the cavity photon. For simplicity, we use a dipole moment that is constant along

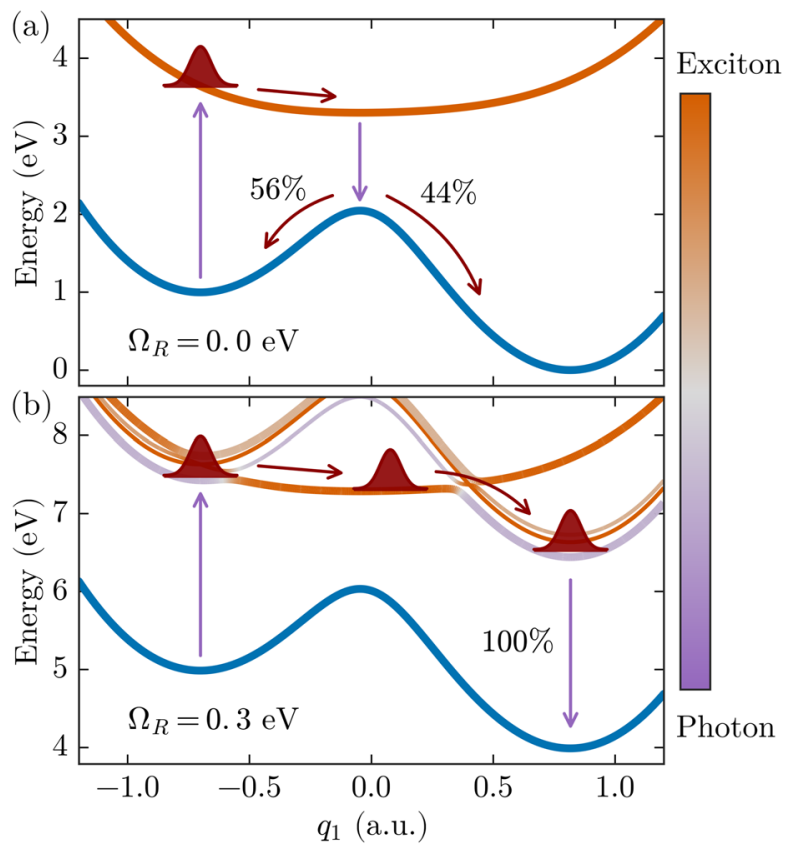

FIG. 1. Potential energy surfaces of (a) the bare molecule and (b) a system with one light mode and $N=5$ molecules in the strong-coupling regime $\left(\Omega_{R}=0.3 \mathrm{eV}\right)$, with all molecules but one fixed at the initial position $\left(q_{1}=q_{\mathrm{ms}} \approx-0.7\right.$ a.u. $)$. The photon energy is $\omega_{c}=2.55 \mathrm{eV}$. The color scale represents the cavity mode fraction of the excited states, going from pure photon (purple) to pure exciton (orange).

the reaction coordinate and assume perfect alignment between the molecular dipoles and the electric field direction. We have checked that these assumptions are not essential, see Sec. II of the Supplemental Material [23] for more details. The electronic and photonic part of the full $N$-molecule Hamiltonian (which determines the hybrid light-matter PES on which nuclear motion takes place) is then given by

$\hat{H}_{\mathrm{SC}}(\vec{q})=\omega_{c} \hat{a}^{\dagger} \hat{a}+\sum_{i}\left[\hat{V}\left(q_{i}\right)+\hat{\vec{\mu}}\left(q_{i}\right) \cdot \vec{E}_{1 \mathrm{ph}}\left(\hat{a}^{\dagger}+\hat{a}\right)\right]$,

where $\hat{V}$ is the diagonal electronic potential operator with the previously calculated PESs $V_{g}(q)$ and $V_{e}(q)$, while $\vec{E}_{1 \mathrm{ph}}$ is the single-photon electric field, and $\hat{a}^{\dagger}$ and $\hat{a}$ are the bosonic creation and annihilation operators associated with the confined light mode of energy $\omega_{c}$. Diagonalization of $\hat{H}_{\mathrm{SC}}$ then yields the polaritonic PES. The collective coupling strength can be parametrized through the collective Rabi frequency $\Omega_{R}=2 \sqrt{N} \vec{\mu}_{\text {eg }} \cdot \vec{E}_{1 \mathrm{ph}}$, which we assume to be constant as $N$ changes. This corresponds to varying the photonic mode volume $V$ (which determines the singlephoton field strength $\vec{E}_{1 \mathrm{ph}} \propto V^{-1 / 2}$ ) while keeping the molecular density $N / V$ constant. In the following, we focus on the lowest few excited PESs, which to a very good approximation contain a single photonic or electronic 
excitation (as long as the ultra-strong-coupling regime is not reached, i.e., $\Omega_{R} \ll \omega_{c}$ ).

For the motion of just a single molecule, our results already show that the quantum yield for the energy-releasing backreaction can be significantly enhanced under strong coupling. The lowest-energy excited PES [see Fig. 1(b)] is formed by hybridization of the uncoupled excited-state surfaces of the molecules with the surface representing a photon in the cavity and the molecule in the ground state (a copy of the ground-state surface shifted upwards by the photon energy of the confined light mode). The photon energy $\left(\omega_{c}=2.55 \mathrm{eV}\right)$ is close to resonant with the electronic excitation energy at the metastable configuration $\left(q=q_{\mathrm{ms}}\right)$, while most other molecular configurations (and, specifically, the stable configuration $q=q_{\mathrm{s}}$ ) are out of resonance with the cavity. This implies that the nature of the lowest excited-state PES changes depending on the molecular position $q$, corresponding to a polariton in some cases, and corresponding to a bare molecular state in others [as indicated by the color scale in Fig. 1(b)]. In the polaritonic states, each molecule is in its electronic ground state most of the time (since the excitation is distributed over all the molecules and the photonic mode), such that the polaritonic parts of the lowest excited-state PES inherit their shape mostly from the ground-state PES [10]. This leads to the formation of a new minimum in the lowest excited PES at the same position as the fully relaxed ground-state minimum $q_{\mathrm{s}}$. As we have shown previously and demonstrate for the current model in Sec. III of the Supplemental Material [23], this effect can be used to strongly suppress photochemical reactions as it can induce a significant activation barrier [10]. However, for the photon energy $\omega_{c}$ and Rabi frequency $\Omega_{R}$ considered here, no such barrier against vibrational relaxation within the lowest excited-state PES exists. Instead, the PES consists of two polaritonic regions (close to $q_{1}=q_{\mathrm{ms}}$ and $q_{1}=q_{\mathrm{s}}$ ) connected by an almost purely excitonic "bridge" (around $q_{1}=0$, where the cavity-exciton detuning is large), with smooth transitions between these parts. In the absence of barriers, a molecular system will quickly relax to the lowest-energy vibrational state on the lowest excitedstate PES according to Kasha's rule [33]. Large-scale molecular dynamics calculations have recently shown that this rule also applies in polaritonic chemistry [14,34]. Vibrational relaxation in the lowest excited hybrid lightmatter PES will thus lead to localization of the nuclear wave packet close to the ground-state minimum $q_{\mathrm{s}}$. As mentioned above, we assume that nonadiabatic couplings in the bare molecule are negligible along the reaction path, such that the dominant relaxation pathway is radiative decay. For the vibrationally relaxed wave packet at $q_{1} \approx q_{\mathrm{s}}$, this would give a quantum yield of essentially unity for the backreaction from the metastable to the stable configuration. While this already presents a large cavity-induced change of the photochemical properties of such molecules, we next show that the collective nature of the polaritons can result in an even more dramatic qualitative change that allows the system to keep releasing energy, with all molecules relaxing from the metastable to the stable configuration one after the other.

To understand this, we have to take into account that the polaritonic PESs formed under strong coupling encompass the nuclear degrees of freedom of all involved molecules $[9,10]$. This collective nature can in particular also allow nuclear motion on different molecules to become coupled, and in the current case creates a reaction path along which the system can release the energy stored in all molecules, while staying on a single adiabatic PES reached by singlephoton absorption in the initial state. This is demonstrated for motion of two of the involved molecules in Fig. 2(a), which shows a two-dimensional cut of the PES of the lowest-energy excited state (with all other molecules again frozen in the metastable position $q=q_{\mathrm{ms}}$ ). We calculate the minimum energy path (MEP) connecting the initial configuration $q_{1}=q_{2}=q_{\mathrm{ms}}$ to the location where the first two molecules have released their stored energy $\left(q_{1}=q_{2}=q_{\mathrm{s}}\right)$ using the nudged-elastic-band method [35]. This approximate classical trajectory defines the reaction coordinate of the full supermolecule system. The initial position, for
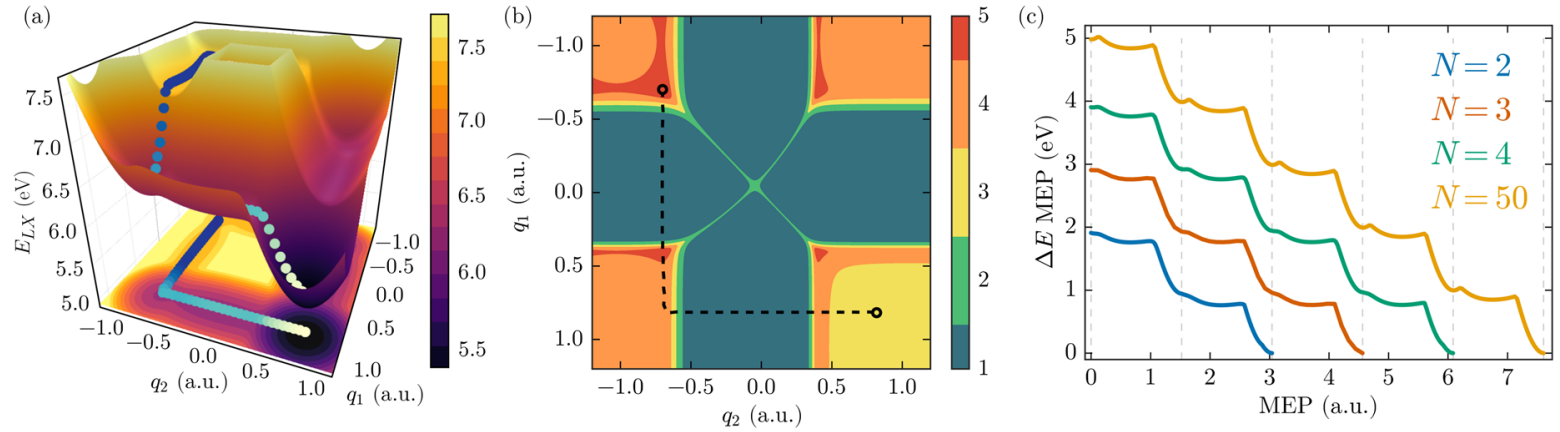

FIG. 2. (a) Lowest-energy excited state PES for two moving molecules in a five-molecule ensemble. The minimum energy path (blue to white dots) connects the initial excited region with the final configuration of the two molecules. (b) Participation ratio map of the lowestenergy excited state, indicating over how many molecules the state is delocalized. The MEP is indicated by a dashed black line. (c) Energy profile along the minimum energy paths for collections of 2, 3, 4, and 50 molecules. For $N=50$, only the first five steps are shown explicitly. Thin dashed lines indicate the approximate location along the path where one molecule stops moving and the next one starts. 
which we assume that all molecules are at $q=q_{\mathrm{ms}}$, corresponds to short-pulse excitation from the ground state in the metastable configuration, according to the FranckCondon principle. The final position is $q_{i}=q_{\mathrm{s}}$ for all $i$, i.e., the position where all the molecules are in the stable configuration (corresponding to the global minimum of the PES). It is worth noticing that due to the indistinguishability of our molecules, any of the available molecules can undergo the reaction in each step, and there are $N$ ! equivalent paths from the initial to the final position. Because of rapid decoherence through interaction with the vibrational bath, we assume that quantum interference between these equivalent paths can be neglected, and we show only one of them in the following, specifically, the one in which the order of reactions corresponds to the numbering of the molecules.

Along this path, indicated as a series of points in Fig. 2(a), there are no significant reaction barriers, such that vibrational relaxation after absorption of a single photon indeed can lead to deactivation of both molecules. The MEP also demonstrates that, to a good approximation, the reaction proceeds in steps, with the molecules moving one after the other (i.e., in the first leg, only $q_{1}$ changes, while in the second leg, only $q_{2}$ changes).

In order to gain additional insight into the properties of the polariton states that enable this stepwise manymolecule reaction triggered by a single photon, we further analyze the lowest excited PES by showing its molecular participation ratio in Fig. 2(b). Here, the molecular participation ratio is defined as [36]

$$
P_{\alpha}(\vec{q})=\frac{\left[\sum_{i}\left|\left\langle e_{i} \mid \Psi_{\alpha}(\vec{q})\right\rangle\right|^{2}\right]^{2}}{\sum_{i}\left|\left\langle e_{i} \mid \Psi_{\alpha}(\vec{q})\right\rangle\right|^{4}},
$$

where $\left|e_{i}\right\rangle$ denotes the excited state of molecule $i$, and the sums are over all molecules. The participation ratio gives an estimate of the number of molecular states that possess a significant weight in a given state $\left|\Psi_{\alpha}\right\rangle$, with possible values ranging from $P_{\alpha}=1$ to $P_{\alpha}=N$ (for $N$ molecules). Analyzing it for the lowest-energy excited-state PES [see Fig. 2(b)] demonstrates that the surface at the starting point corresponds to a collective polariton, with the excitation equally distributed over all molecules. Along the MEP, the excitation collapses onto a single molecule (the one that is moving), demonstrated by the participation ratio decreasing to 1 for -0.5 a.u. $\lesssim q_{1} \lesssim 0.4$ a.u.. As the molecule moves, it again enters into resonance with the cavity (and the other molecules) and the state changes character to a fully delocalized polariton with $P_{L X}=N$ (at $q_{1} \approx 0.45$ a.u.). However, as the first molecule keeps moving, it falls out of resonance again and effectively "drops out" of the polaritonic state, leaving the excitation in a polaritonic state distributed over the photonic mode and the remaining $N-1$ molecules $\left(P_{L X}=4\right)$, which then forms the starting point for the second molecule to undergo the reaction. Following the MEP along the second leg (where $q_{1} \approx q_{\mathrm{s}}$ and $q_{2}$ moves from $q_{\mathrm{ms}}$ to $q_{\mathrm{s}}$ ), the same process repeats, but now involving one less molecule.

We now demonstrate that the same process can keep repeating for many molecules. To this end, we calculate the MEP for varying numbers of molecules from $N=2$ to $N=50$, with a collective Rabi splitting of $\Omega_{R}=0.3 \mathrm{eV}$ in the initial molecular configuration $\left(q_{i}=q_{\mathrm{ms}}\right.$ for all $\left.i\right)$ for all cases. As shown in Fig. 2(c), the energy profile along the MEP is structurally similar for any number of molecules. The main change is that for larger values of $N$, the collective protection effect demonstrated in [10] makes the PES resemble the shape of the uncoupled PES more strongly, leading to a less-smooth MEP with slightly higher barriers, comparable to the average thermal kinetic energy at room temperature. In addition, the significant change of collective state when passing the barrier (with the excitation collapsing from all molecules onto a single one in the "bridge" region around $q=0$ ) leads to narrow avoided crossings in the adiabatic picture. In a adiabatic picture formed by the polaritonic PES of $N-1$ coupled molecules and the remaining bare excited PES, their coupling can be shown to be proportional to the single-molecule coupling strength $\Omega_{R} / \sqrt{N}$ [10], such that to lowest order in perturbation theory, the transition probability from the polaritonic to the pure-exciton PES scales as $1 / N$. However, this effect is compensated by the fact that there are many possible equivalent paths, corresponding to motion of any of the remaining metastable molecules. We thus assume that transition state theory (TST) for a single barrier of the same height provides a reasonable estimate for the average time needed to overcome any one of the barriers. This gives $\tau \approx$ $\left(h / k_{B} T\right) \exp \left(\Delta E / k_{B} T\right) \lesssim 1$ ps at room temperature [22]. It should be noted that several other mechanisms relevant in the current case imply that the TST predictions correspond to an upper limit, as TST is known to fail in multistep reactions where the nuclear wave packet approaches the barriers with some initial kinetic energy [37], and also neglects quantum tunneling effects that are important for small energy barriers as found here.

The estimated times for passing the barriers highlight the importance of the lifetime of the hybrid light-matter states to determine the feasibility of triggering multiple reactions with a single photon. In most current experiments, polariton lifetimes (which are an average of the lifetimes of their constituents) are very short, on the order of tens of femtoseconds, due to the use of short-lived photonic modes such as localized surface plasmons or low- $Q$ modes in metallic and dielectric Fabry-Perot microcavities. In contrast, the lifetime of the molecular excitations can be limited by their spontaneous radiative decay, which is on the order of nanoseconds for typical organic molecules. Consequently, if long-lived photonic modes as available in low-loss dielectric structures such as photonic crystals or microtoroidal cavities are used instead, there is no fundamental reason preventing polariton lifetimes that approach nanoseconds. This would thus give enough time 
for thousands of molecules to undergo a reaction before the excitation is lost due to radiative decay.

In conclusion, we have demonstrated that under strong coupling, a single photon could be used to trigger a photochemical reaction in many molecules. This corresponds to an effective quantum yield (number of reactant molecules per absorbed photon) of the reaction that is significantly larger than 1 , and thus provides a possible pathway to break the second (or Stark-Einstein) law of photochemistry without relying on fine-tuned resonance conditions. The basic physical effect responsible for this surprising feature is the delocalized nature of the polaritonic states obtained under collective strong coupling, which require a treatment of the whole collection of molecules as a single polaritonic supermolecule. For the specific model studied here, this strategy could resolve one of the main problems of solar energy storage; that is, how to efficiently retrieve the stored energy from molecules that are designed for the opposite purpose, i.e., for storing energy very efficiently under normal conditions [20,21]. By reversibly bringing the system into strong coupling (e.g., through a moving mirror that brings the cavity into and out of resonance), one could thus trigger the release of the stored energy through absorption of a single ambient photon.

We thank G. Groenhof for helpful discussions. This work has been funded by the European Research Council under Grants No. ERC-2011-AdG-290981 and No. ERC2016-STG-714870, by the European Union Seventh Framework Programme under Grant No. FP7-PEOPLE2013-CIG-618229, and the Spanish MINECO under Contract No. MAT2014-53432-C5-5-R and the "María de Maeztu" program for Units of Excellence in R\&D (MDM-2014-0377).

*fj.garcia@uam.es †johannes.feist@uam.es

[1] K. K. Rohatgi-Mukherjee, Fundamentals of Photochemistry (New Age International, New Delhi, India, 2013), p. 370.

[2] D. P. Summers, J. C. Luong, and M. S. Wrighton, A new mechanism for photosubstitution of organometallic complexes. Generation of substitutionally labile oxidation states by excited-state electron transfer in the presence of ligands, J. Am. Chem. Soc. 103, 5238 (1981).

[3] T. Arai, T. Karatsu, H. Sakuragi, and K. Tokumari, "Oneway" photoisomerization between cis- and trans-olefin. A novel adiabatic process in the excited state, Tetrahedron Lett. 24, 2873 (1983).

[4] B. J. Eves, Q.-Y. Sun, G. P. Lopinski, and H. Zuilhof, Photochemical attachment of organic monolayers onto H-terminated Si(111): Radical chain propagation observed via STM studies, J. Am. Chem. Soc. 126, 14318 (2004).

[5] B. J. Walker, A. J. Musser, D. Beljonne, and R. H. Friend, Singlet exciton fission in solution, Nat. Chem. 5, 1019 (2013).
[6] J. Zirzlmeier, D. Lehnherr, P. B. Coto, E. T. Chernick, R. Casillas, B. S. Basel, M. Thoss, R. R. Tykwinski, and D. M. Guldi, Singlet fission in pentacene dimers, Proc. Natl. Acad. Sci. U.S.A. 112, 5325 (2015).

[7] J. A. Hutchison, T. Schwartz, C. Genet, E. Devaux, and T. W. Ebbesen, Modifying chemical landscapes by coupling to vacuum fields, Angew. Chem. 124, 1624 (2012).

[8] S. Wang, A. Mika, J. A. Hutchison, C. Genet, A. Jouaiti, M. W. Hosseini, and T. W. Ebbesen, Phase transition of a perovskite strongly coupled to the vacuum field, Nanoscale 6, 7243 (2014).

[9] J. Galego, F. J. Garcia-Vidal, and J. Feist, Cavity-Induced Modifications of Molecular Structure in the StrongCoupling Regime, Phys. Rev. X 5, 041022 (2015).

[10] J. Galego, F. J. Garcia-Vidal, and J. Feist, Suppressing photochemical reactions with quantized light fields, Nat. Commun. 7, 13841 (2016).

[11] F. Herrera and F. C. Spano, Cavity-Controlled Chemistry in Molecular Ensembles, Phys. Rev. Lett. 116, 238301 (2016).

[12] M. Kowalewski, K. Bennett, and S. Mukamel, Nonadiabatic dynamics of molecules in optical cavities, J. Chem. Phys. 144, 054309 (2016).

[13] T. W. Ebbesen, Hybrid light-matter states in a molecular and material science perspective, Acc. Chem. Res. 49, 2403 (2016).

[14] S. Baieva, O. Hakamaa, G. Groenhof, T. T. Heikkilä, and J. J. Toppari, Dynamics of strongly coupled modes between surface plasmon polaritons and photoactive molecules: The effect of the Stokes shift, ACS Photonics 4, 28 (2017).

[15] J. Flick, M. Ruggenthaler, H. Appel, and A. Rubio, Atoms and molecules in cavities, from weak to strong coupling in quantum-electrodynamics (QED) chemistry, Proc. Natl. Acad. Sci. U.S.A. 114, 3026 (2017).

[16] J. Flick, H. Appel, M. Ruggenthaler, and A. Rubio, Cavity Born-Oppenheimer approximation for correlated electron-nuclear-photon systems, J. Chem. Theory Comput. 13, 1616 (2017).

[17] M. Kowalewski and S. Mukamel, Manipulating molecules with quantum light, Proc. Natl. Acad. Sci. U.S.A. 114, 3278 (2017).

[18] L. A. Martínez-Martínez, R. F. Ribeiro, J. Campos-GonzálezAngulo, and J. Yuen-Zhou, Can ultrastrong coupling change ground-state chemical reactions?, arXiv:1705.10655.

[19] T. J. Kucharski, Y. Tian, S. Akbulatov, and R. Boulatov, Chemical solutions for the closed-cycle storage of solar energy, Energy Environ. Sci. 4, 4449 (2011).

[20] M. Cacciarini, A. B. Skov, M. Jevric, A. S. Hansen, J. Elm, H. G. Kjaergaard, K. V. Mikkelsen, and M. Brøndsted Nielsen, Towards solar energy storage in the photochromic dihydroazulene-vinylheptafulvene system, Chem. Eur. J. 21, 7454 (2015).

[21] J. Gurke, M. Quick, N. P. Ernsting, and S. Hecht, Acidcatalysed thermal cycloreversion of a diarylethene: A potential way for triggered release of stored light energy? Chem. Commun. (Cambridge) 53, 2150 (2017).

[22] H. Eyring, The activated complex in chemical reactions, J. Chem. Phys. 3, 107 (1935).

[23] See Supplemental Material at http://link.aps.org/ supplemental/10.1103/PhysRevLett.119.136001, which includes Refs. [24,25], for details on the bare-molecule 
quantum yields, the molecular model, and suppression of the reaction when choosing slightly different cavity parameters.

[24] V. Bonačić-Koutecký, P. Bruckmann, P. Hiberty, J. Koutecký, C. Leforestier, and L. Salem, Sudden polarization in the zwitterionic Z1 excited states of organic intermediates. Photochemical implications, Angew. Chem. 14, 575 (1975).

[25] B. G. Levine and T. J. Martínez, Isomerization through conical intersections, Annu. Rev. Phys. Chem. 58, 613 (2007).

[26] S. M. Spillane, T. J. Kippenberg, and K. J. Vahala, Ultralowthreshold Raman laser using a spherical dielectric microcavity, Nature (London) 415, 621 (2002).

[27] Y. Akahane, T. Asano, B.-S. Song, and S. Noda, High-Q photonic nanocavity in a two-dimensional photonic crystal, Nature (London) 425, 944 (2003).

[28] K. S. Daskalakis, S. A. Maier, R. Murray, and S. KénaCohen, Nonlinear interactions in an organic polariton condensate, Nat. Mater. 13, 271 (2014).

[29] P. Lodahl, S. Mahmoodian, and S. Stobbe, Interfacing single photons and single quantum dots with photonic nanostructures, Rev. Mod. Phys. 87, 347 (2015).

[30] G. Zengin, M. Wersäll, S. Nilsson, T. J. Antosiewicz, M. Käll, and T. Shegai, Realizing Strong Light-Matter Interactions Between Single-Nanoparticle Plasmons and
Molecular Excitons at Ambient Conditions, Phys. Rev. Lett. 114, 157401 (2015).

[31] R. Chikkaraddy, B. de Nijs, F. Benz, S. J. Barrow, O. A. Scherman, E. Rosta, A. Demetriadou, P. Fox, O. Hess, and J. J. Baumberg, Single-molecule strong coupling at room temperature in plasmonic nanocavities, Nature (London) 535, 127 (2016).

[32] M. Ramezani, A. Halpin, A. I. Fernández-Domínguez, J. Feist, S. R.-K. Rodriguez, F. J. Garcia-Vidal, and J. Gómez Rivas, Plasmon-exciton-polariton lasing, Optica 4, 31 (2017).

[33] M. Kasha, Characterization of electronic transitions in complex molecules, Discuss. Faraday Soc. 9, 14 (1950).

[34] H. L. Luk, J. Feist, J. J. Toppari, and G. Groenhof, Multiscale molecular dynamics simulations of polaritonic chemistry, J. Chem. Theory Comput., DOI: 10.1021/ acs.jctc.7b00388 (2017).

[35] G. Henkelman, B. P. Uberuaga, and H. Jónsson, A climbing image nudged elastic band method for finding saddle points and minimum energy paths, J. Chem. Phys. 113, 9901 (2000).

[36] B. Kramer and A. MacKinnon, Localization: Theory and experiment, Rep. Prog. Phys. 56, 1469 (1993).

[37] E. V. Anslyn and D. A. Dougherty, Modern Physical Organic Chemistry (University Science Books, Sausalito, California, 2006), p. 1136. 